Proceedings of the 43rd "Jaszowiec", International School and Conference on the Physics of Semiconductors, Wisła 2014

\title{
Desorption of Gasses Induced by Ferroelectric Transition in SbSI Nanowires
}

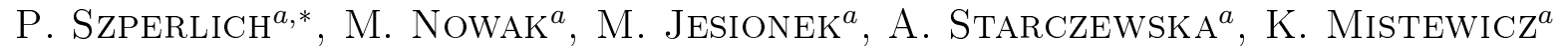 \\ AND J. SZALA ${ }^{b}$ \\ ${ }^{a}$ Solid State Physics Section, Institute of Physics - Center for Science and Education, \\ Silesian University of Technology, Z. Krasińskiego 8, 40-019 Katowice, Poland \\ ${ }^{b}$ Department of Materials Science, Silesian University of Technology, Z. Krasińskiego 8, 40-019 Katowice, Poland
}

For the first time the thermal desorption of $\mathrm{H}_{2}, \mathrm{~N}_{2}, \mathrm{O}_{2}$ and $\mathrm{CO}_{2}$ is presented for antimony sulfoiodide (SbSI) xerogel made up of large quantity nanowires. The desorption has been observed near ferroelectric phase transition established at $T_{\mathrm{c}}=293.0(2) \mathrm{K}$. The Sievert measurements have shown that the hydrogen uptake is linear function of $\mathrm{H}_{2}$ pressure (when $\mathrm{p}<1.1 \times 10^{5} \mathrm{~Pa}$ ). The hydrogen storage density in SbSI gel amounted $1.24 \times 10^{-2} \mathrm{wt} \%$ (for $\mathrm{p}=1.08 \times 10^{5} \mathrm{~Pa}$ at room temperature).

DOI: $10.12693 /$ APhysPolA.126.1110

PACS: 68.43.Vx, 77.84.-s, 68.43.-h, 62.23.Hj

\section{Introduction}

Ferroelectrics contain electric dipoles that are intrinsic to their atomic structure. Orientation of the dipoles in ferroelectrics influences adsorption of polar or polarized molecules [1]. The changes in local dipole orientation and its permanent moment affect the strength of the coupling between adsorbate and the ferroelectric material. While in ferroelectric phase transition the spontaneous polarization changes, the anomalies of adsorption or desorption are expected [2].

Recent experiments have proved the strong interaction of the ferroelectric antimony sulfoiodide (SbSI) gel with the water dipoles $[3-5]$. The aim of this paper was to study the influence of ferroelectric transition on desorption of different gasses from high-surface-area SbSI xerogel. This material has large surface-to-volume ratio. It was found that SbSI nanocrystals amounts only $4.7 \%$ of the xerogel volume [6].

\section{Experiment}

SbSI was synthesized sonochemically from the constituents (the elements $\mathrm{Sb}, \mathrm{S}$ and I) weighed in the stoichiometric ratio. Application of ultrasounds in chemistry has the following advantages: evokes short reaction time, changes conditions necessary for chemical reactions (e.g., decreases temperature of the process) increases chemical activity of reacting substances, causes higher productivity. It happens due to acoustic cavitation: creation, growth and collapse of bubbles that are formed in the liquid. Details of the applied procedure, used experimental setup, and characterization of the obtained material were presented in [5]. After sonication, product

*corresponding author; e-mail: piotr.szperlich@polsl.pl of synthesis was dried in air at room temperature. As the liquid was evaporated, so-called SbSI xerogel was obtained (Fig. 1a). Figure 1b presents a typical scanning electron microscopy (SEM) micrograph of the sonochemically prepared SbSI that was taken on Hitachi S-4200 scanning electron microscope. This material exists as a porous xerogel composed of nanowires with average lateral dimensions of 10-50 $\mathrm{nm}$ and average lengths reaching up to several $\mu \mathrm{m}$.

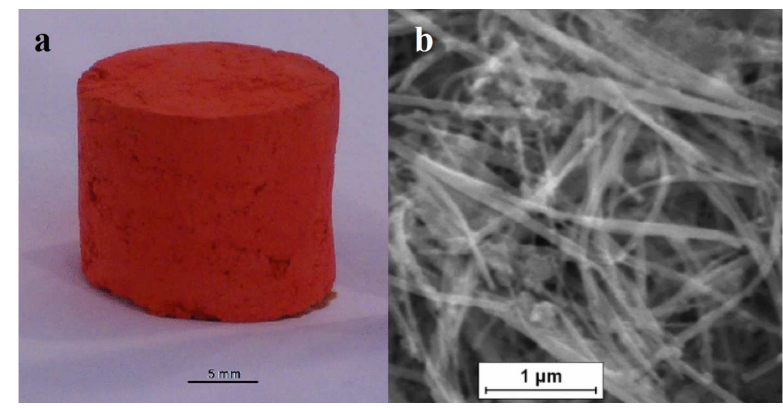

Fig. 1. Photograph (a) and typical SEM micrograph (b) of SbSI gel obtained by the sonochemical method.

For ac electric measurements, sample was cut from SbSI xerogel into cuboid with dimensions: $3.20(1) \mathrm{mm}$ $\times 5.02(1) \mathrm{mm} \times 2.41(1) \mathrm{mm}$. The largest opposite surfaces of the sample were covered with silver paste (SPI Supplies). The capacity measurements were performed at $1 \mathrm{kHz}$ using HIOKI 3532-50 LCR meter in air at atmospheric pressure. To monitor temperature of the sample, Pt-100 sensor was placed near to the investigated sample and connected to the 211 temperature monitor (Lake Shore). The temperature dependence of the dielectric constant of the SbSI gel is shown in Fig. 2.

In order to perform thermal desorption spectroscopy (TDS), SbSI gel was put in vacuum chamber equipped 




Fig. 2. Temperature dependence of the averaged dielectric constant of SbSI gel $(\mathrm{E}=4.15 \mathrm{kV} / \mathrm{m}, \mathrm{f}=1 \mathrm{kHz}$, $\mathrm{p}=10^{5} \mathrm{~Pa}, \mathrm{RH}=73 \%$ ). Solid curve represents the Curie-Weiss relation calculated for the best fitted $\mathrm{C}=$ $1.41(14) \times 10^{4} \mathrm{~K}$ and $\mathrm{T}_{\mathrm{C}}=293.0(2) \mathrm{K}$.

with TW70H turbomolecular drag pumping station (Prevac) and vacuum gauges ACC 1009, ADS 1001 with ACM 1000 controller (Alcatel). The composition of gas atmosphere in chamber was controlled using quadrupole mass spectrometer UMS 200 (Prevac) with residual gas analyzer RGA200 (Stanford Research Systems). The setup for temperature measurement were the same as in the case of electrical investigations. Before TDS examinations, the sample was annealed and outgassed in vacuum $\left(\mathrm{p}=10^{-1} \mathrm{~Pa}\right)$ at $333 \mathrm{~K}$ for $1 \mathrm{~h}$ to clean the surface. After that, sample was cooled down to room temperature. Then the high purity gas $\left(\mathrm{H}_{2}, \mathrm{O}_{2}, \mathrm{~N}_{2}\right.$ or $\left.\mathrm{CO}_{2}\right)$ was introduced into the vacuum chamber using a needle valve (Leybold EV016 DOSAB) to maintain the desired gas pressure of about $10^{5} \mathrm{~Pa}$. The sample was kept under this gas for 5 min at room temperature. Afterward, SbSI xerogel was cooled down and kept in $77 \mathrm{~K}$ for $30 \mathrm{~min}$. Finally, the gas was pumped out and the sample was heated with a nearly linear temperature rate $(6 \mathrm{~K} / \mathrm{min})$ up to $323 \mathrm{~K}$. The heating was carried out with continuous evacuation of gas. Figure 3a displays TDS spectra obtained using pressure $(p)$ measurements after exposing SbSI xerogel to different gasses.

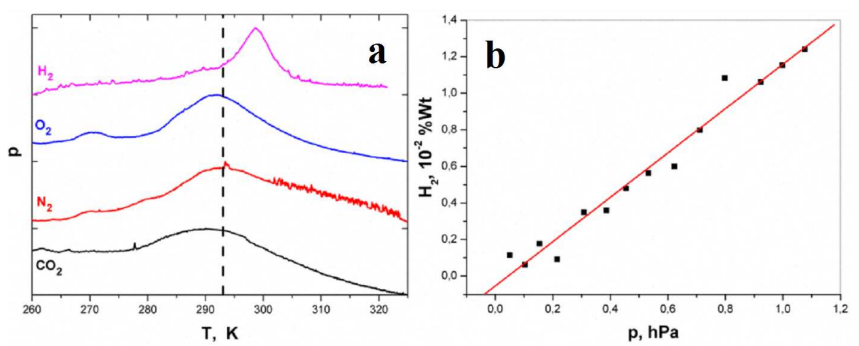

Fig. 3. (a) TDS spectra obtained after dosing SbSI gel with different gasses and (b) hydrogen storage density in SbSI gel as a function of gas pressure $(\mathrm{T}=300 \mathrm{~K})$. The vertical dashed line shows the temperature of ferroelectric transition in the investigated SbSI nanowires.
The hydrogen adsorption studies of SbSI gel were carried out at room temperature using Sievert's method, in the pressure range from $5 \times 10^{3} \mathrm{~Pa}$ to $1.1 \times 10^{5} \mathrm{~Pa}$. In this method, a calibrated reference volume is filled with gas to a measured pressure and then opened to the sample chamber, the gas uptake by the sample being calculated from the change in the gas pressure in the system [7]. Hydrogen was allowed at the temperature of $300 \mathrm{~K}$. Equipment for temperature and pressure measurements were exactly the same as in the case of TDS investigations.

\section{Discussion}

The surface area of the sonochemically prepared SbSI is about $75 \mathrm{~m}^{2} / \mathrm{g}[6]$. It is consistent with the SEM observations (Fig. 1b). The extremely high surface-tovolume ratios associated with these nanostructures make their properties extremely sensitive to species adsorbed on surfaces.

The temperature dependence of the dielectric constant of sonochemically prepared SbSI gel proves that this material is a ferroelectric with the Curie temperature $\left(T_{\mathrm{C}}\right)$ near $293 \mathrm{~K}$ (Fig. 2). Such Curie temperature is characteristic for SbSI single crystals [8]. For temperatures above $T_{\mathrm{C}}, \varepsilon(\mathrm{T})$ dependence was least-squares fitted (Fig. 2) with the Curie-Weiss relation with the Curie constant $\mathrm{C}=1.41(14) \times 10^{4} \mathrm{~K}$ and the Curie temperature $\mathrm{T}_{\mathrm{C}}=293.0(2) \mathrm{K}$. The determined maximum value $1.46 \times 10^{4}$ of dielectric constant is little lower than the peak $\varepsilon\left(\mathrm{T}_{\mathrm{C}}=292(1) \mathrm{K}\right)=1.6 \times 10^{4}$ reported in [9] for SbSI ethanogel and less than the $\varepsilon\left(\mathrm{T}_{\mathrm{C}}=291 \mathrm{~K}\right)=6.2 \times 10^{4}$ measured along the polar axis of the best SbSI single crystals [10]. One should remember that SbSI is a highly anisotropic material and the permittivity of SbSI single crystal of the polar direction is about 2000 larger than that of the perpendicular direction [11]. It should be underlined that the investigated SbSI xerogel has very large porosity so the determined averaged value of dielectric constant does not represent the exact value for the SbSI nanowires.

As shown in Fig. 3a, desorption of each of the investigated gas attains well-defined maximum in the pressuretemperature graph. All the gases reveal desorption near the $\mathrm{T}_{\mathrm{C}}$. The observed discrepancies in the temperatures of the maximum desorption can be explained by the wellknown influence of adsorbates on temperature of phase transitions in dielectrics [12]. It was demonstrated for an ultrathin $\mathrm{PbTiO}_{3}$ film [13], that the chemical environment can even control the polarization orientation in a ferroelectric layer. It should be underlined that the shift of phase transition temperature, caused by the adsorption of gas molecules, was used [12] to create a highly sensitive gas pressure sensors made of bulk SbSI.

Adsorption and desorption of gasses on SbSI xerogel may be explained as follows. The dipole moment of a polar molecule (or induced dipole moment) interacts with the electric polarization of some ferroelectric domains at the surface. Gas-phase molecules become trapped into a 
shallow physisorption well, dominated by Van der Waals interactions and influenced by polarization of the ferroelectrics [1]. Substantial depression of the ferroelectricity should decrease the amount of adsorbed molecules. The energy of desorption should correlate with the value of spontaneous polarization. Since the physisorbed precursor bonds to the surface primarily through Van der Waals dipole-dipole interactions, the polarity of the adsorbing species would also be expected to have an effect.

The presented in Fig. 3b results of Sievert's measurements have revealed that hydrogen storage density in SbSI gel is a linear function of $\mathrm{H}_{2}$ pressure (for $\left.p<1.1 \times 10^{5} \mathrm{~Pa}\right)$. The proportionality factor equals $1.21(6) \times 10^{-7} \% / \mathrm{Pa}$. The hydrogen storage capacity of $1.24 \times 10^{-2}$ wt\% of SbSI gel body weight was achieved for $\mathrm{p}=1.08 \times 10^{5} \mathrm{~Pa}$ at room temperature. Unfortunately, it is too small to be practical for hydrogen storage. However, it should be underlined that many data on hydrogen uptake in nanomaterials were registered for $\mathrm{H}_{2}$ pressure equal or higher than $10^{7} \mathrm{~Pa}$ [14]. The observed (Fig. 3b) linearly proportionality of hydrogen uptake of SbSI gel to the gas pressure is very promising. The hydrogen storage in low temperature should be much higher. Hence, the appropriate investigations are necessary in the future.

\section{Conclusions}

The ferroelectric phase transition in SbSI xerogel is accompanied by activation of the thermal desorption of atoms and molecules. This effect could have technological implications in the areas of gas storage and sensitive gas sensors.

The hydrogen uptake in SbSI gel is linearly proportional to the pressure (for $\mathrm{p}<1.1 \times 10^{5} \mathrm{~Pa}$ ). The hydrogen storage density in SbSI gel has reached up to $1.24 \times 10^{-2} \mathrm{wt} \%$ for $\mathrm{p}=1.08 \times 10^{5} \mathrm{~Pa}$ at room temperature. It should be interesting to determine the adsorption of $\mathrm{H}_{2}$ in low temperature and under high pressure.

\section{Acknowledgments}

This paper was partially supported by the National Science Centre project no. DEC-2011/01/B/ST5/06273.

\section{References}

[1] D. Li, M.H. Zhao, J. Garra, A.M. Kolpak, A.M. Rappe, D.A. Bonnell, J.M. Vohs, Nature Mater. 7, 473 (2008).

[2] A.L. Cabrera, F. Vargas, R.A. Zarate, J. Phys. Chem. Solids 55, 1303 (1994).

[3] A. Starczewska, M. Nowak, P. Szperlich, B. Toron, K. Mistewicz, D. Stróż, J. Szala, Sensor. Actuat. A-Phys. 183, 34 (2012).

[4] M. Nowak, K. Mistewicz, A. Nowrot, P. Szperlich, M. Jesionek, A. Starczewska, Sensor. Actuat. A-Phys. 210, 32 (2014).

[5] M. Nowak, A. Nowrot, P. Szperlich, M. Jesionek, M. Kępińska, A. Starczewska, K. Mistewicz, D. Stróż, J. Szala, T. Rzychoń, E. Talik, R. Wrzalik, Sensor. Actuat. A-Phys. 210, 119 (2014).

[6] M. Nowak, P. Szperlich, Ł. Bober, J. Szala, G. Moskal, D. Stróż, Ultrason. Sonochem. 15, 709 (2008).

[7] T.P. Blach, E.MacA. Grey, J. Alloys Comp. 446447, 692 (2007).

[8] K. Toyoda, Ferroelectrics 69, 201 (1986).

[9] P. Szperlich, M. Nowak, Ł. Bober, J. Szala, D. Stróż, Ultrason. Sonochem. 16, 398 (2009).

[10] K. Ishikawa, W. Tomoda, K. Toyoda, J. Cryst. Growth 69, 399 (1984).

[11] W.J. Merz, R. Nitsche, Izv. Akad. Nauk SSSR Ser. Fiz. 28, 681 (1964).

[12] Yu.V. Popik, V.N. Zhikharev, V.V. Betsa, Fiz. Tverd. Tela 24, 486 (1982).

[13] R.V. Wang, D.D. Fong, F. Jiang, M.J. Highland, P.H. Fuoss, C. Thompson, A.M. Kolpak, J.A. Eastman, S.K. Streiffer, A.M. Rappe, G.B. Stephenson, Phys. Rev. Lett. 102, 047601 (2009).

[14] M.M. Shaijumon, S. Ramaprabhu, Chem. Phys. Lett. 374, 513 (2003) 